\title{
Review Article \\ Bone Regulates Glucose Metabolism as an Endocrine Organ through Osteocalcin
}

\author{
Jin Shao, Zhi Wang, Tieyi Yang, Hui Ying, Yan Zhang, and Shuyi Liu \\ Department of Orthopaedics, Shanghai Pudong New Area Gongli Hospital/Clinical School, The Second Military Medical University, \\ Shanghai 200135, China \\ Correspondence should be addressed to Tieyi Yang; yangtieyi@163.com
}

Received 9 June 2014; Revised 21 August 2014; Accepted 18 September 2014

Academic Editor: Andrea Del Fattore

Copyright (C) 2015 Jin Shao et al. This is an open access article distributed under the Creative Commons Attribution License, which permits unrestricted use, distribution, and reproduction in any medium, provided the original work is properly cited.

\begin{abstract}
Skeleton was considered as a dynamic connective tissue, which was essential for mobility, calcium homeostasis, and hematopoietic niche. However more and more evidences indicate that skeleton works not only as a structural scaffold but also as an endocrine organ, which regulates several metabolic processes. Besides osteoprotegerin (OPG), sclerostin (SOST), and Dickopf (DKK) which play essential roles in bone formation, modelling, remodelling, and homeostasis, bone can also secret hormones, such as osteocalcin (OCN), which promotes proliferation of $\beta$ cells, insulin secretion, and insulin sensitivity. Additionally OCN can also regulate the fat cells and male gonad endocrine activity and be regulated by insulin and the neural system. In summary, skeleton has endocrine function via OCN and plays an important role in energy metabolism, especially in glucose metabolism.
\end{abstract}

\section{Introduction}

Bone has been only considered as a structure organ for centuries. Classical understanding of skeleton is that bone functions as the structure scaffold in animals and contains three types of cells: osteoblast, osteocyte, and osteoclast. Osteoblasts come from mesenchymal stem cells and can differentiate to osteocytes, which stay in the bone matrix. Osteoblasts are responsible for bone formation, and its activity can be regulated by several pathways such as WNT signaling pathway. Osteoclasts are a type of bone cells, which resorb bone and derived from the fusion of mononuclear cells belonging to the monocyte/macrophage lineage. Osteocytes and osteoblasts can secrete some signaling factors, such as WNT, OPG, and SOST, in order to regulate the coupling function of osteoblast and osteoclast; in this way bone obtains the ability to model and remodel itself. Recent reports indicate that SOST, a WNT antagonist, downregulates the WNT signaling activity by crosstalk with parathyroid hormone (PTH) and bone morphogenetic protein (BMP) in order to regulate bone cell differentiation, proliferation, bone formation, and bone resorption activities [1-4]. Even though there are plenty of evidences indicating that bone can secrete protein factors in the circulation and regulate itself, all these secreted factors cannot be called hormones.

However, in the past decades with the development of technology we found that skeleton can also be an endocrine organ, which regulates not only itself but also other organs. The most well known and studied two hormones secreted by skeleton are OCN and fibroblast growth factor 23 (FGF23) [5] (Figure 1). In this review we would focus on OCN, especially on its function as a hormone in metabolism homeostasis.

\section{Endocrine Functions of Bone through OCN}

2.1. Production of OCN. OCN is specially synthesized and secreted by cells of osteoblast lineage, such as osteoblast and osteocyte $[6,7]$. In humans $\mathrm{OCN}$ is also known as bone $\gamma$-carboxyglutamic acid-containing protein (BGP) and it is one of the richest noncollagenous proteins in bone matrix. The most of OCN is found in bone matrix and only a small amount in blood, because it has strong affinity to bone matrix.

OCN has proosteoblastic or bone-building function. Osteoblasts can secret $\mathrm{OCN}$ to stimulate osteoblastic differentiation and osteocytic maturation. The OCN gene has 


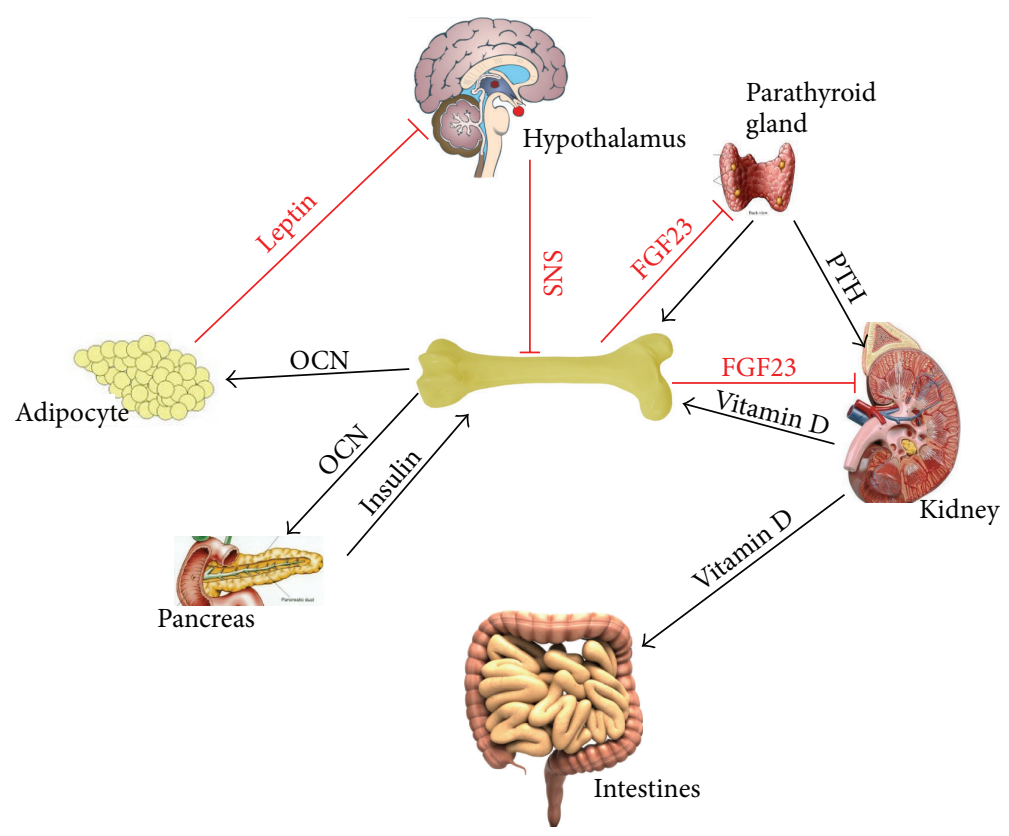

Figure 1: Skeleton regulates mineral and energy homeostasis. In mineral homeostasis, low level of circulating calcium stimulates the parathyroid gland to release PTH, which later upregulates blood calcium levels by stimulating osteoclastic bone resorption, renal calcium reabsorption, and renal production of Vitamin D to increase intestinal calcium absorption. Increased serum phosphate and Vitamin D stimulate FGF23 synthesis and releasing in bone, which subsequently inhibits PTH production from the parathyroid gland, inhibits Vitamin D production in the kidney, and promotes renal phosphate excretion. Leptin inhibits bone formation and the homeostatic function of the skeleton indirectly through SNS. However, SNS signalling also increases the production of OCN from bone, which feeds into the positive loop. OCN affects pancreatic $\beta$ cells and increases insulin level, which has feedback effect on bone, driving further production of OCN. OCN also acts on fat to increase the production of adiponectin and upregulates insulin sensitivity. FGF23, fibroblast growth factor 23; PTH, parathyroid hormone; SNS, sympathetic nervous system.

4 exons and 3 introns, and more than $70 \%$ of the exon sequences are conserved both in human and in mice. The OCN protein contains 46-50 amino acid residues (varying from different species), the synthesis process of which is a little complicated (Figure 2). Firstly, the transcription is regulated by $1 \alpha, 25$-dihydroxy-Vitamin D3. After the translation it is only preproosteocalcin, which contains 98 amino acid residues composed of three parts: a 23-residue signal protein, a 26-residue target propeptide, and a 49-residue mature protein. Proteolysis of the prepropeptide will form the mature OCN [8]. At position 17, 21 and 24 of the 49residue peptide second carboxyl groups $(-\mathrm{COOH})$ are added to form $\gamma$-carboxyglutamyl residues, which are essential for its activity regulation.

According to the carboxylation level, OCN can be divided into two groups; one is called undercarboxylated osteocalcin ( $\mathrm{ucOCN})$, and the other is carboxylated osteocalcin (cOCN). The decarboxylated form ucOCN is the active form of OCN as a hormone, which has little affinity to bone so that most of ucOCN will circulate with the blood, while most of $\mathrm{cOCN}$ will be stored in the bone matrix because of its strong affinity to bone matrix. Usually the concentration of ucOCN is not controlled by the protein synthesis, but by the decarboxylation of OCN and its releasing from the bone matrix. This issue will be discussed in Section 2.3 later.

\subsection{OCN Targeting Organs}

2.2.1. OCN Targets $\beta$ Cells and Adipocytes. There are evidences indicating that in Ocn knocked out mice the total amount of insulin in serum is downregulated, and the Ocn knocked out mice have so-called impaired glucosestimulated insulin secretion (GSIS) and poor glucose tolerance phenotype [9]. At the same time the serum adiponectin, a protein hormone that modulates several metabolic processes such as glucose regulation and fatty acid oxidation, was also reduced [10]. All these results suggest that OCN may target $\beta$ cells and insulin targeting tissue such as muscle, liver, and adipocyte in order to regulate both insulin secretion and sensitivity [11, 12]. Later studies show that the change of insulin sensitivity is probably mediated by adiponectin instead of direct interaction with OCN [13]. This result indicates that adipocytes are probably one target of $\mathrm{ucOCN}$, and ucOCN functions as the activity form of OCN in circulation.

There are also many evidences indicating that ucOCN can regulate $\beta$ cells in pancreatic islets. Not only insulin 1 and insulin 2 can be upregulated by ucOCN but also the proliferation of $\beta$ cells. Further research indicates that CyclinD1, CyclinD2, and Cdk 4 in $\beta$ cells can also be regulated by ucOCN, and in this way it is not difficult to understand why ucOCN can stimulate $\beta$ cell proliferation $[9,12,14]$. 


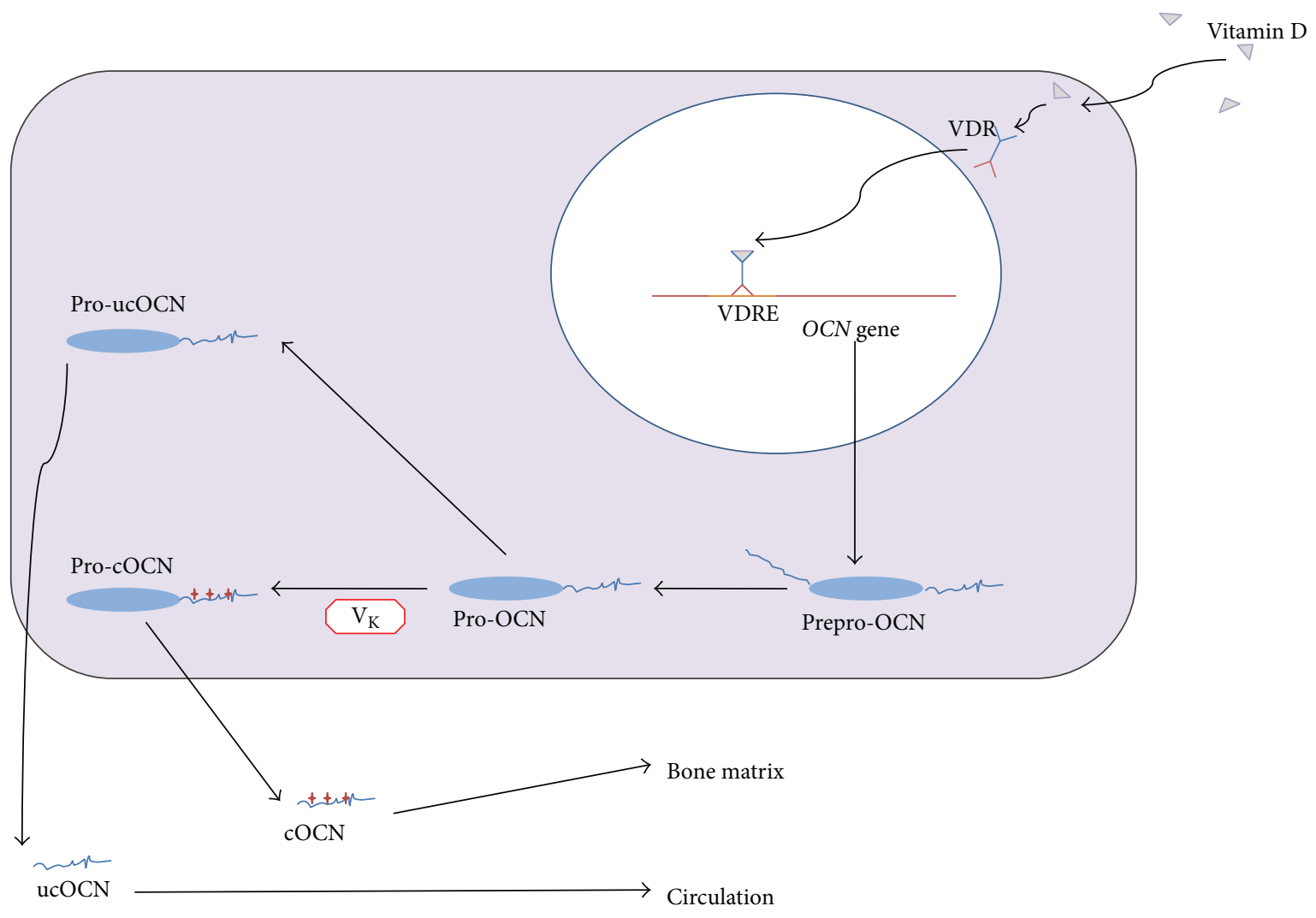

FIGURE 2: OCN is synthesized in osteoblasts. OCN is mainly expressed in osteoblasts. After transcription, which is stimulated by Vitamin D, the prepro-OCN peptide will be proteolysed and forms a prepeptide ( $23 \mathrm{aa})$ and a pro-OCN peptide (75 aa). The pro-OCN peptide can be carboxylated at Glu residues 17, 21, and 24, resulting in formation of Gla residues in a Vitamin K dependent process. Generally, this process only occurs in a proportion of newly synthesized proosteocalcin. Then Gla and Glu pro-OCN peptides are subjected to a final proteolytic process that produces $\mathrm{COCN}$ and ucOCN. Both forms are released from osteoblasts in a process which is calcium dependent. While the carboxylated Gla residues are involved in calcium and hydroxyapatite binding, allowing OCN deposition on mineralized bone matrix, ucOCN has a low affinity for hydroxyapatite and is more easily released into the circulation. OCN, osteocalcin; ucOCN, uncarboxylated osteocalcin; VDR, Vitamin D receptor; VDRE, Vitamin D receptor element.

$\beta$ cells proliferation and insulin secretion can be significantly affected at low concentrations of ucOCN (6-60 pM) [15]. The mice lacking osteoblasts have not only problems on bone density and strength but also impaired glucose metabolism, such as high blood glucose, low insulin secretion, and insulin resistance. All these phenotypes mimic the defects in $\mathrm{Ocn}$ knocked out mice. However, administration of OCN can restore glucose and insulin level in circulation but only partially insulin sensitivity [16]. This indicates that osteoblasts may have some OCN independent ways to regulate insulin sensitivity.

Although more and more evidences show that OCN can regulate $\beta$ cells and increase the insulin sensitivity of insulin targeting cells, the receptor of OCN or more correctly ucOCN has not been identified yet. What is more, we do not even know how OCN regulates the insulin sensitivity. There are only evidences indicating adiponectin mediates the insulin sensitivity regulation of OCN [13]. Whether some other mechanisms involved in this progress is still not clear. We should also not ignore $\mathrm{COCN}$, which usually stays in the bone matrix. The biological function of $\mathrm{COCN}$ is still unclear. Is it only storage of ucOCN? Does it have some unknown functions on metabolism of bone formation? Some in vitro studies indicated that $\mathrm{COCN}$ could regulate bone formation and resorption; however, in the Ocn knocked out mice no negative bone effects were observed [17-19]. Anyway, a lot of questions still need to be answered.

2.2.2. OCN Regulates Testicular Function. OCN regulates male reproduction activity [20]. These were observed from the experimental studies on Esp and Ocn knocked out mice. With or without OCN, male mice showed different reproductive activity. Mice with high $\mathrm{OCN}$ activity (the Esp knocked out mice) had increased testicular volume and sperm count. On the contrary, the $\mathrm{OCN}$ absent mice (the $\mathrm{Ocn}$ knocked out mice) showed shriveled testes, epididymis, and seminal vesicles. However, the female in both Esp and $O c n$ knocked out groups did not show any defects in reproduction. Ocn specific deletion in leydig cells showed no such effects as described above [20]. This indicates that OCN secreted by skeleton regulates male reproduction activity as a hormone.

And fortunately the receptor in the leydig cell of ucOCN was identified. It is a $G$ protein-coupled receptor (GPCR6A) 


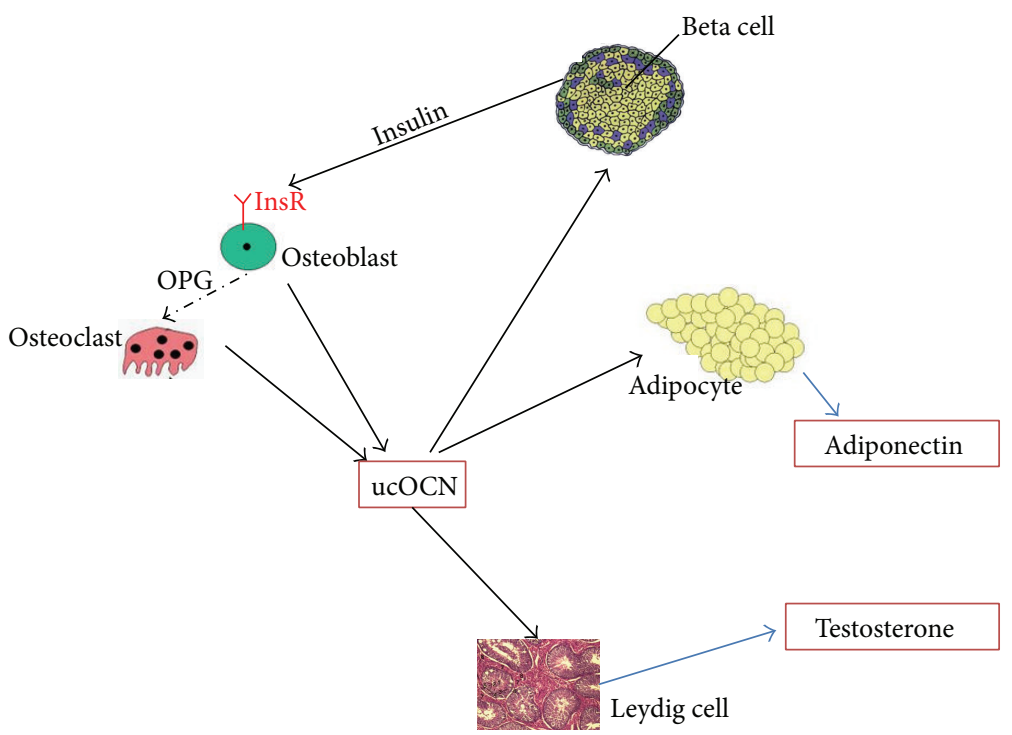

FIGURE 3: Endocrine actions of osteocalcin. Circulating OCN and particularly ucOCN (released during bone resorption activity) have direct effects on $\beta$ cells, stimulating insulin synthesis. Meanwhile it affects adipocytes and stimulates adiponectin secretion. Adiponectin upregulates insulin sensitivity. In turn, insulin binds to insulin receptor of osteoblasts and affects osteoblasts; then OPG expression will be upregulated and in turn suppress the activity of osteoclasts. Osteoclast stimulates bone resorption with subsequent release of ucOCN in blood circulation. OCN also functions on leydig cells. It can increase their activity and testosterone production. OCN, osteocalcin; ucOCN, uncarboxylated osteocalcin; OPG, osteoprotegerin; InsR, insulin receptor.

and localized on the surface of the leydig cell. This 7transmembrane protein was found because the GPCR6A knocked out mice copy the metabolic syndrome of the Ocn knocked out mice [21, 22]. In the past decades it was demonstrated that gonadal hormone (estrogen) can regulate bone formation $[23,24]$, and now we know that bone can also regulate testicle and testosterone secretion. This discovery suggested that there may be a novel mechanism regulating bone formation in some special stage, such as teenage time, in which rapid bone formation is associated with androgen secretion changing. In general, $\mathrm{OCN}$, particularly ucOCN (released during bone resorption) targets directly $\beta$ cells and stimulates insulin synthesis and secretion. At the same time it affects adipocytes and enhances adiponectin secretion. Adiponectin itself upregulates the insulin sensitivity of the insulin targeting cells [13]. Interestingly, OCN also targets leydig cells and regulates testosterone production and male reproductive activity (Figure 3 ).

\subsection{Regulation of OCN Production and Activation}

2.3.1. OCN Is Partly Regulated by 1 $\alpha, 25-D i h y d r o x y v i t a m i n$ D3 and Vitamin K. Vitamin D is a kind of fat-soluble secosteroids which plays an essential role in absorption of calcium, phosphorus, iron, zinc, and their metabolism. In humans and mice the most active form of Vitamin $D$ is $1 \alpha, 25$-dihydroxyvitamin D3 (Vitamin D3). Recent studies showed that the Ocn expression in osteoblasts can be partly stimulated by Vitamin D3 [25]. It has been reported that OCN usually functions to inhibit mineralization. More interestingly fibroblast growth factor 23 (FGF23), another bone secreted hormone, can affect kidney and inhibit Vitamin D
TABLE 1: Metabolic phenotypes of ESP and Ocn knocked out mice.

\begin{tabular}{lcc}
\hline & $\triangle E S P$ mice & $\Delta O c n$ mice \\
\hline Blood glucose & Decreased & Increased \\
Glucose tolerant & Increased & Decreased \\
Sensitive to insulin & Increased & Decreased \\
Beta cells proliferation & Increased & Decreased \\
ucOCN & Decreased & Absent \\
\hline
\end{tabular}

synthesis. This loop suggested a feedback loop, in which bone can partly regulate its own hormone secretion [26].

Studies showed that accumulation of osteocalcin in the ECM of human osteoblastic cultures stimulated by $1 \alpha, 25-$ dihydroxyvitamin D3 is inhibited by warfarin (antagonist of Vitamin K), while Vitamin K2 enhanced the $1 \alpha$,25-dihydroxyvitamin D3 effect [27], and 1 $\alpha, 25$-dihydroxyvitamin D3 stimulated mineralization was significantly augmented by warfarin [28].

The biological active form of $\mathrm{OCN}$ is $\mathrm{ucOCN}$, while the cOCN has a strong affinity to bone matrix and binds tightly to hydroxyapatite, as a result little cOCN could be found in the serum [29]. Vitamin $\mathrm{K}$ is a cofactor for the glutamate carboxylase, which is required for carboxylation. In the absence of Vitamin $\mathrm{K}$ the serum level of ucOCN would be increased. On the contrary, high level of Vitamin $\mathrm{K}$ can reduce ucOCN level, which had been proved by Vitamin $\mathrm{K}$ administration in daily diet [30].

2.3.2. OST-PTP, the Product of Receptor-Type Tyrosine-Protein Phosphatase V (Esp) Controls OCN Activity. Generally speaking, there are two ways controlling OCN signaling, one is 


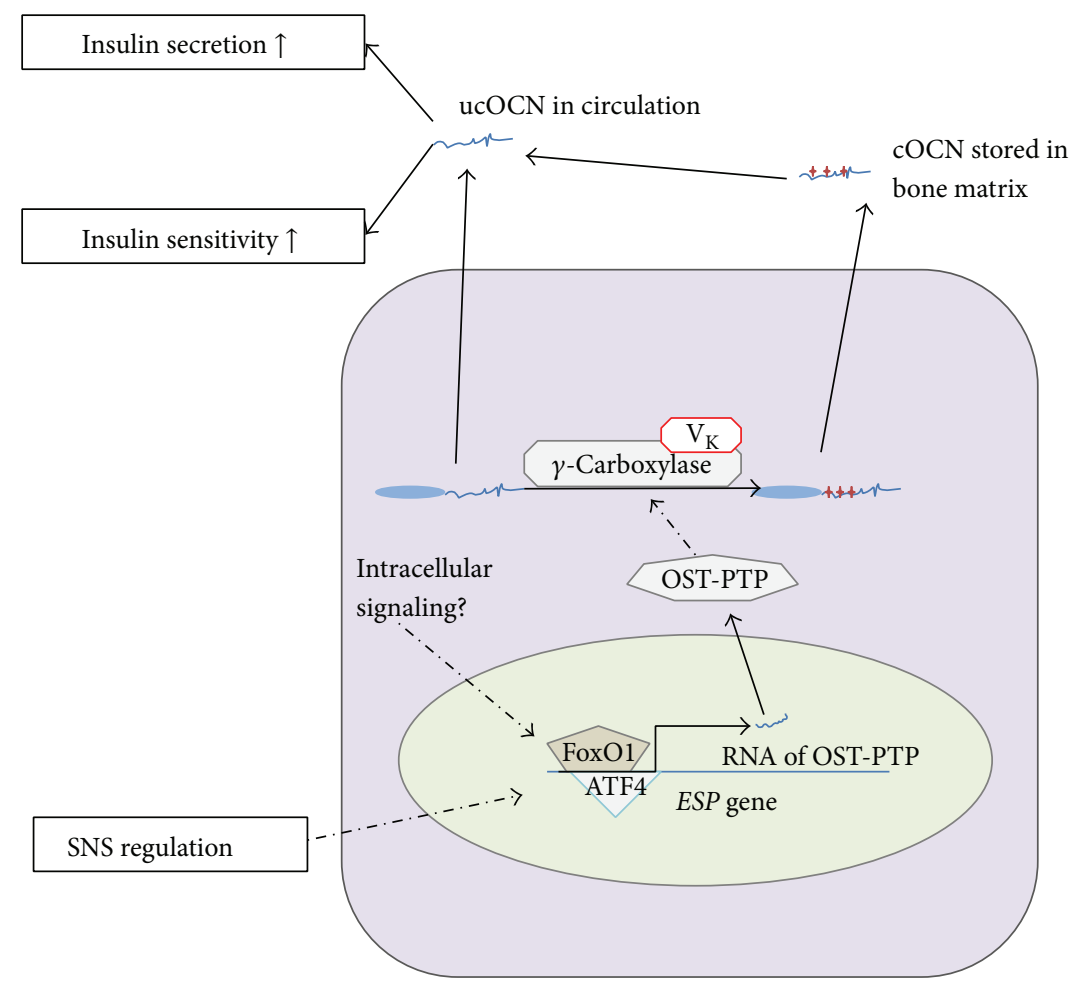

FIGURE 4: Proposed regulation of Esp expression and OCN carboxylation in mice. FoxO1 and ATF4 could bind to the promoter site to stimulate the transcription of Esp. The product of Esp is OST-PTP, which can suppress the carboxylation of prepro-OCN and in turn suppress the activity of OCN. $\mathrm{V}_{\mathrm{K}}$, Vitamin K; SNS, sympathetic nervous system; OCN, osteocalcin; ucOCN, uncarboxylated osteocalcin.

on the transcription level and the other is on the posttranslational level. The product of Esp is an osteotesticular protein tyrosine phosphatase (OST-PTP), which is found in embryonic cells, Sertoli cells, and osteoblast [9]. Karsenty et al. found Esp knocked out mice have the opposite phenotype compared with the Ocn knocked out group (Table 1). The phenotypes of the Esp knocked out mice are as follows: hypoglycemia, high glucose-stimulated insulin secretion, glucose tolerance, pancreatic insulin content, and $\beta$ cell proliferation [9]. Esp deletion (either totally knocked out or selectively knocked out in osteoblasts) resulted in the exact opposite phenotype as found in the Ocn knocked out mice. And Esp can rescue the phenotype of the high fat diet and gold thioglucose-induced obesity and diabetes, which mimics the phenotype of ucOCN treated mice $[9,12]$. All these results let Lee et al. believe that OST-PTP may mediate the inactivation of OCN via $\gamma$-carboxylation. Indeed lacking one allele of Ocn in the Esp knocked out mice rescued the metabolic abnormality of the Esp absent mice. This result indicates that OST-PTP downregulates the activity of ucOCN [9]. As the total Ocn gene expression did not change in Esp knocked out mice, Ferron et al. suggested that OST-PTP was involved in the osteoclast-dependent ucOCN formation and releasing [31].

In the recent years the molecular mechanism of Esp regulation was revealed (Figure 4). Two transcription factors, forkhead box protein O1 (FOXO1) and cyclic
AMP-dependent transcription factor (ATF-4), are involved in the regulation of the total OCN [32]. FOXO1 is a conservative modulator of glucose metabolism in many organs, as well as insulin synthesis in $\beta$ cells, and ATF4 regulates expression of many genes which mostly are important for adult bone formation and development $[33,34]$. Osteoblast specific FoxO1 knocked out mice have low blood glucose level and high glucose tolerance [35], which is similar to the mice with high ucOCN level. This indicated that FoxO1 may be a modulator of $\mathrm{Ocn}$ transcription or posttranslational modification. Further studies on knocked out mice showed that FoxO1 could upregulate OST-PTP. Molecular experimental studies showed that FoxO1 could bind to a cognate FoxO1 binding site in the Esp promoter area and stimulate the expression of the downstream Esp; then OST-PTP would activate the carboxylation of OCN or ucOCN. The new formed cOCN would bind to hydroxyapatite and stay in bone matrix, and as a result the ucOCN would be downregulated [35]. These studies also found the binding site for ATF4 in the Esp promoter. Now it is not surprising that ATF4 can suppress obesity in mice and involve in the glucose homeostasis in human [36-38]. In fact even though ATF4 is mainly expressed in osteoblast, it functions quite similar to FoxO1. Via stimulating OST-PTP transcription ATF4 suppress the activity of OCN, in this way suppressing insulin secretion and insulin sensitivity $[39,40]$. More details of OCN regulation 
are still unknown, and the endocrine regulation of OCN will be discussed in the later section of this review.

\section{Bone Regulates Glucose Metabolism via $\mathrm{OCN}$}

3.1. OCN in Glucose Metabolism. As mentioned in the sections above, in the Ocn knocked out mice the blood glucose concentration was higher than that of the wild type. Serum insulin in the knocked out mice would be downregulated and the mice tended to be obesity [12]. All these indicate that OCN may be essential in energy metabolism, especially in glucose metabolism. With administration of OCN or deletion of Esp (OST-PTP increases ucOCN, the active form of OCN), the mice achieved higher activity of insulin, lower blood glucose, higher glucose tolerance, and higher insulin sensitivity with normal glucagon levels [9]. This abnormality is mainly caused by the increased number or mass of pancreatic islets and $\beta$ cells. This means that $\mathrm{OCN}$ can increase insulin secretion and insulin sensitivity in some tissues such as muscles and adipose tissue. The increasing sensitivity of insulin is probably mediated by adiponectin, which can be upregulated by ucOCN $[9,12,41]$. cOCN would be stored in the bone matrix and released during bone resorption [10,42]. Osteoclasts would secret protons and decrease the $\mathrm{pH}$ value during resorption; this would facilitate the decarboxylation of $\mathrm{COCN}$ and by the way the active form ucOCN would enter the circulation to stimulate $\beta$ cells proliferation and insulin secretion $[31,43]$. However, more details of the mechanism need to be revealed.

3.2. Feed-Forward Effect of Insulin. Insulin, synthesized and modified in $\beta$ cells in pancreas, is an essential protein hormone in almost all vertebrates. Through clinical observation we know that insulin signaling can also affect the bones. Insulin receptor (InsR) is required for osteoblast survival, proliferation, and differentiation [44, 45]. Runx2 is a transcription factor which is essential for osteoblastic differentiation and skeletal morphogenesis. Studies showed that the expression of Runx 2 was decreased in osteoblasts lacking InsR $[45,46]$. However, insulin dose does not directly regulate Runx2 activity. Instead, insulin downregulates Twist2, an inhibitor of Runx2 activity [46], to suppress the activity of Runx2 [43].

Insulin can also promote bone resorption. However, it does not directly work on osteoclasts but on osteoblasts. Insulin signaling can decrease osteoprotegerin (OPG) expression in osteoblasts via binding to InsR [31]. OPG, a key regulator of osteoclasts, together with receptor activator of nuclear factor- $\kappa$ ligand (RANKL) via receptor activator of nuclear factor- $\kappa$ (RNAK) regulates the differentiation and activity of osteoclasts $[2,47,48]$. During bone resorption the osteoclasts secrete proton and the $\mathrm{pH}$ value will be low. This acidic environment facilitates decarboxylation and the concentration of ucOCN will be upregulated. In turn ucOCN increases $\beta$ cells proliferation, insulin secretion, and insulin sensitivity $[16,49]$. Thus, insulin signaling and OCN form a feed-forward loop, in which insulin affects osteoblasts and in turn increases its own secretion and sensitivity via ucOCN. Insulin signaling can decrease OPG expression in osteoblast via binding to InsR [31]. And InsR is downstream molecule of OST-PTP, which inhibits phosphorylation of InsR and stops insulin signaling in osteoblasts $[50,51]$. This feed-forward model explains why the osteoblastic InsR knocked out mice have low serum insulin level and high insulin resistance.

3.3. Endocrine Regulation of OCN. As mentioned above, the relationship between OCN and insulin is not a feedback relationship, so there must be some other signaling pathways to regulate the OST-PTP activity. If not, the serum insulin concentration cannot stay in a certain level with only the feed-forward loop between bone and pancreatic islets. Some studies suggested that sympathetic nervous system (SNS) may control the expression of Esp via leptin, a hormone secreted by adipocytes [41]. The main function of leptin is regulating the amount of fat stored in the body. It can adjust both the sensation of hunger and energy expenditures. The target of leptin signaling is the brain. It inactivates the 5hydroxylase 2 (Tph2), which is known as the rate-limiting enzyme of serotonin synthesis [52]. Tph2 knocked out mice have osteoporotic and anorectic phenotype, indicating this source of serotonin can regulate osteoblasts. Yadav et al. have proved that leptin-dependent regulation of OCN carboxylation appeared to be through the hypothalamus $[53,54]$. The SNS acts through $\beta 2$-adrenergic receptors (Adrb2) in osteoblasts to upregulate the Esp expression [41], and in so doing the activity of ucOCN would be suppressed.

\subsection{OCN in Human}

3.4.1. ESP Does Not Play the Same Role in Human. The ESP gene in human is a so-called pseudogene which does not have a functional product. Its function may be replaced by another or some other proteins [55]. Years ago a study showed that in human dephosphorylation of InsR can be achieved by protein-tyrosine phosphatase $1 \mathrm{~B}$ (PTP1B) instead of OSTPTP [31]. So it seems that the mechanism of OCN regulation in human is different from that in mice.

\subsubsection{The Circadian Rhythm of OCN in Human Is Different} from That in Mice. In human the concentration of OCN will arrive at its peak in the early morning and its lowest point in the afternoon, while the mice will have a high level of $\mathrm{OCN}$ in the day and the level of OCN reaches its lowest point at night during sleep [56]. In the older studies the researchers could only test the concentration of total OCN, now there are several ways to test the level of ucOCN, such as ucOCN specific ELISA and hydroxyapatite (HAP) binding assay $[19,57]$. With these technological development, recent studies showed that the ratio of ucOCN/OCN usually did not change, while the total amount of ucOCN in circulation had the similar changes as the circadian rhythm of OCN $[8,58-$ $62]$.

3.4.3. Clinical Studies of OCN. In clinical studies the data were mostly focused on the effects of OCN on bone formation 
and metabolism. Children and adolescents, who have the need to increase bone formation, usually have higher levels of OCN and ucOCN and this may facilitate the utilization of glucose in bone [63]. Clinical studies also showed that bone loss was associated with high level of ucOCN in human, and in older people high OCN level predicted increased fracture risk or lower bone density $[64,65]$. The phenomenon can be explained by studies in mice that bone resorption facilitates decarboxylation of OCN and releases OCN from bone matrix to the blood [10]. However, whether OCN has effects on skeleton or not is still not clear; the OCN knocked out mice seem quite normal. The minor differences between the knocked out and wild type mice suggest that OCN may play some roles in regulation of bone mineralization [17].

Only in recent years many studies are focused on the hormone functions of ucOCN and OCN in energy metabolism. Patients who suffer from type 1 or type 2 diabetes mellitus have lower level of OCN than healthy people [66-69]. Patients with obesity also show lower level of OCN [70, 71]. We have already discussed that adiponectin mediates the insulin sensitivity. However, studies showed in male patients with type 2 diabetes adiponectin was associated with $\mathrm{ucOCN} / \mathrm{OCN}$ ratio instead of the concentration of ucOCN, while in female patients it was correlated with $\mathrm{OCN}$, but not ucOCN [72]. It is now very clear that OCN regulates testicular function and does not have such function on female gonad [20]. Whether it has something to do with the differences between male and female diabetic patients is still unknown. Anyway, more studies need to be done to reveal the mechanism of this phenomenon.

\section{Conclusion}

Studies in animal models showed that ucOCN targets $\beta$ cells in the pancreas to directly regulate insulin synthesis and regulates insulin sensitivity through adiponectin [13]. And experiments in mice showed that ucOCN may be a potential therapy for diabetic patients [66]. One of the regulators of OCN is OST-PTP, which promotes the carboxylation of OCN and reduces the concentration of $\mathrm{uCOCN}$ or the ratio of ucOCN/OCN [8].

There are also many clinical evidences indicating that OCN or probably ucOCN is associated with fasting glucose and insulin sensitivity [63]. However, OST-PTP is not a functional protein in human, and the exact role which OCN plays in human still needs to be studied.

Anyway all these studies suggest us skeleton can behave not only as a structure scaffold but also as an endocrine organ, which regulates energy metabolism, even though all the studies are mostly done in mice and only partially confirmed in human [55]. Therefore, more investigations are needed to reveal the total functions of OCN as a hormone in energy metabolism.

\section{Conflict of Interests}

The authors declare that there is no conflict of interests regarding the publication of this paper.

\section{Authors' Contribution}

Jin Shao and Zhi Wang contributed equally to this work.

\section{Acknowledgments}

This work was supported by National Natural Science Foundation of China (81201367), Key Discipline Construction Project of Pudong Health Bureau of Shanghai (PWZx201409), Academic Leaders Training Program of Pudong Health Bureau of Shanghai (PWRd2012-16), and Shanghai Municipal Natural Science Foundation (10ZR1427700).

\section{References}

[1] R. Baron and M. Kneissel, "WNT signaling in bone homeostasis and disease: from human mutations to treatments," Nature Medicine, vol. 19, no. 2, pp. 179-192, 2013.

[2] S. Khosla, "Minireview: the OPG/RANKL/RANK system," Endocrinology, vol. 142, no. 12, pp. 5050-5055, 2001.

[3] M. E. Brunkow, J. C. Gardner, J. van Ness et al., "Bone dysplasia sclerosteosis results from loss of the SOST gene product, a novel cystine knot-containing protein," The American Journal of Human Genetics, vol. 68, no. 3, pp. 577-589, 2001.

[4] M. Katoh, "Networking of WNT, FGF, Notch, BMP, and Hedgehog signaling pathways during carcinogenesis," Stem Cell Reviews, vol. 3, no. 1, pp. 30-38, 2007.

[5] S. Fukumoto and T. J. Martin, "Bone as an endocrine organ," Trends in Endocrinology and Metabolism, vol. 20, no. 5, pp. 230236, 2009.

[6] P. V. Hauschka, J. B. Lian, D. E. Cole, and C. M. Gundberg, "Osteocalcin and matrix Gla protein: vitamin K-dependent proteins in bone," Physiological Reviews, vol. 69, no. 3, pp. 990 1047, 1989.

[7] P. A. Price, "Gla-containing proteins of bone," Connective Tissue Research, vol. 21, no. 1-4, pp. 51-57, 1989.

[8] A. J. Lee, S. Hodges, and R. Eastell, "Measurement of osteocalcin," Annals of Clinical Biochemistry, vol. 37, part 4, pp. 432-446, 2000.

[9] N. K. Lee, H. Sowa, E. Hinoi et al., "Endocrine regulation of energy metabolism by the skeleton," Cell, vol. 130, no. 3, pp. 456469, 2007.

[10] K. K. Ivaska, T. A. Hentunen, J. Vääräniemi, H. Ylipahkala, K. Pettersson, and H. K. Väänänen, "Release of intact and fragmented osteocalcin molecules from bone matrix during bone resorption in vitro," The Journal of Biological Chemistry, vol. 279, no. 18, pp. 18361-18369, 2004.

[11] I. Kanazawa and T. Sugimoto, "The relationship between bone and glucose/lipid metabolism," Clinical Calcium, vol. 23, no. 2, pp. 181-188, 2013.

[12] M. Ferron, E. Hinoi, G. Karsenty, and P. Ducy, "Osteocalcin differentially regulates $\beta$ cell and adipocyte gene expression and affects the development of metabolic diseases in wild-type mice," Proceedings of the National Academy of Sciences of the United States of America, vol. 105, no. 13, pp. 5266-5270, 2008.

[13] A. S. Lihn, S. B. Pedersen, and B. Richelsen, "Adiponectin: action, regulation and association to insulin sensitivity," Obesity Reviews, vol. 6, no. 1, pp. 13-21, 2005.

[14] G. L. Klein, "Insulin and bone: recent developments," World Journal of Diabetes, vol. 5, no. 1, pp. 14-16, 2014. 
[15] S. L. Booth, A. Centi, S. R. Smith, and C. Gundberg, "The role of osteocalcin in human glucose metabolism: marker or mediator?" Nature Reviews Endocrinology, vol. 9, no. 1, pp. 4355, 2013.

[16] Y. Yoshikawa, A. Kode, L. Xu et al., "Genetic evidence points to an osteocalcin-independent influence of osteoblasts on energy metabolism," Journal of Bone and Mineral Research, vol. 26, no. 9, pp. 2012-2025, 2011.

[17] A. L. Boskey, S. Gadaleta, C. Gundberg, S. B. Doty, P. Ducy, and G. Karsenty, "Fourier transform infrared microspectroscopic analysis of bones of osteocalcin-deficient mice provides insight into the function of osteocalcin," Bone, vol. 23, no. 3, pp. 187-196, 1998.

[18] T. Reinehr and C. L. Roth, "A new link between skeleton, obesity and insulin resistance: relationships between osteocalcin, leptin and insulin resistance in obese children before and after weight loss," International Journal of Obesity, vol. 34, no. 5, pp. 852-858, 2010.

[19] P. Vergnaud, P. Garnero, P. J. Meunier, G. Bréart, K. Kamihagi, and P. D. Delmas, "Undercarboxylated osteocalcin measured with a specific immunoassay predicts hip fracture in elderly women: the EPIDOS study," Journal of Clinical Endocrinology and Metabolism, vol. 82, no. 3, pp. 719-724, 1997.

[20] F. Oury, G. Sumara, O. Sumara et al., "Endocrine regulation of male fertility by the skeleton," Cell, vol. 144, no. 5, pp. 796-809, 2011.

[21] M. Pi, L. Chen, M.-Z. Huang et al., "GPRC6A null mice exhibit osteopenia, feminization and metabolic syndrome," PLoS ONE, vol. 3, no. 12, Article ID e3858, 2008.

[22] M. Pi and L. D. Quarles, "Multiligand specificity and wide tissue expression of GPRC6A reveals new endocrine networks," Endocrinology, vol. 153, no. 5, pp. 2062-2069, 2012.

[23] Y. Miyauchi, Y. Sato, T. Kobayashi et al., "HIFlalpha is required for osteoclast activation by estrogen deficiency in postmenopausal osteoporosis," Proceedings of the National Academy of Sciences of the United States of America, vol. 110, no. 41, pp. 16568-16573, 2013.

[24] X. Dai, C. Wang, J. Dai et al., "Association of single nucleotide polymorphisms in estrogen receptor alpha gene with susceptibility to knee osteoarthritis: a case-control study in a Chinese Han population," BioMed Research International, vol. 2014, Article ID 151457, 12 pages, 2014.

[25] J. van de Peppel and J. P. T. M. van Leeuwen, "Vitamin D and gene networks in human osteoblasts," Frontiers in Physiology, vol. 5, article 137, Article ID Article 137, 2014.

[26] D. J. DiGirolamo, T. L. Clemens, and S. Kousteni, “The skeleton as an endocrine organ," Nature Reviews Rheumatology, vol. 8, no. 11, pp. 674-683, 2012.

[27] Y. Koshihara and K. Hoshi, "Vitamin $\mathrm{K}_{2}$ enhances osteocalcin accumulation in the extracellular matrix of human osteoblasts in vitro," Journal of Bone and Mineral Research, vol. 12, no. 3, pp. 431-438, 1997.

[28] V. J. Woeckel, C. Bruedigam, M. Koedam, H. Chiba, B. C. J. van der Eerden, and J. P. T. M. van Leeuwen, " $1 \alpha, 25-$ Dihydroxyvitamin D3 and rosiglitazone synergistically enhance osteoblast-mediated mineralization," Gene, vol. 512, no. 2, pp. 438-443, 2013.

[29] J. R. Cairns and P. A. Price, "Direct demonstration that the vitamin K-dependent bone Gla protein is incompletely $\gamma$ carboxylated in humans," Journal of Bone and Mineral Research, vol. 9, no. 12, pp. 1989-1997, 1994.
[30] L. J. Sokoll and J. A. Sadowski, "Comparison of biochemical indexes for assessing vitamin $\mathrm{K}$ nutritional status in a healthy adult population," American Journal of Clinical Nutrition, vol. 63, no. 4, pp. 566-573, 1996.

[31] M. Ferron, J. Wei, T. Yoshizawa et al., "Insulin signaling in osteoblasts integrates bone remodeling and energy metabolism," Cell, vol. 142, no. 2, pp. 296-308, 2010.

[32] S. Kousteni, "FoxO1, the transcriptional chief of staff of energy metabolism," Bone, vol. 50, no. 2, pp. 437-443, 2012.

[33] R. T. Franceschi, C. Ge, G. Xiao, H. Roca, and D. Jiang, "Transcriptional regulation of osteoblasts," Cells Tissues Organs, vol. 189, no. 1-4, pp. 144-152, 2008.

[34] W. Wang, N. Lian, L. Li et al., "Atf4 regulates chondrocyte proliferation and differentiation during endochondral ossification by activating Ihh transcription," Development, vol. 136, no. 24, pp. 4143-4153, 2009.

[35] M.-T. Rached, A. Kode, B. C. Silva et al., "FoxO1 expression in osteoblasts regulates glucose homeostasis through regulation of osteocalcin in mice," Journal of Clinical Investigation, vol. 120, no. 1, pp. 357-368, 2010.

[36] M. Delépine, M. Nicolino, T. Barrett, M. Golamaully, G. Mark Lathrop, and C. Julier, "EIF2AK3, encoding translation initiation factor 2- $\alpha$ kinase 3, is mutated in patients with WolcottRallison syndrome," Nature Genetics, vol. 25, no. 4, pp. 406-409, 2000.

[37] H. P. Harding, H. Zeng, Y. Zhang et al., "Diabetes mellitus and exocrine pancreatic dysfunction in Perk-/- mice reveals a role for translational control in secretory cell survival," Molecular Cell, vol. 7, no. 6, pp. 1153-1163, 2001.

[38] D. Ron and P. Walter, "Signal integration in the endoplasmic reticulum unfolded protein response," Nature Reviews Molecular Cell Biology, vol. 8, no. 7, pp. 519-529, 2007.

[39] T. Yoshizawa, E. Hinoi, Y. J. Dae et al., "The transcription factor ATF4 regulates glucose metabolism in mice through its expression in osteoblasts," Journal of Clinical Investigation, vol. 119, no. 9, pp. 2807-2817, 2009.

[40] A. Kode, I. Mosialou, B. C. Silva et al., "FoxO1 protein cooperates with ATF4 protein in osteoblasts to control glucose homeostasis," Journal of Biological Chemistry, vol. 287, no. 12, pp. 8757-8768, 2012.

[41] E. Hinoi, N. Gao, D. Y. Jung et al., "The sympathetic tone mediates leptin's inhibition of insulin secretion by modulating osteocalcin bioactivity," Journal of Cell Biology, vol. 183, no. 7, pp. 1235-1242, 2008.

[42] C. M. Gundberg and R. S. Weinstein, "Multiple immunoreactive forms of osteocalcin in uremic serum," Journal of Clinical Investigation, vol. 77, no. 6, pp. 1762-1767, 1986.

[43] K. Fulzele, R. C. Riddle, D. J. DiGirolamo et al., "Insulin receptor signaling in osteoblasts regulates postnatal bone acquisition and body composition," Cell, vol. 142, no. 2, pp. 309-319, 2010.

[44] J. C. Brüning, M. D. Michael, J. N. Winnay et al., "A musclespecific insulin receptor knockout exhibits features of the metabolic syndrome of NIDDM without altering glucose tolerance," Molecular Cell, vol. 2, no. 5, pp. 559-569, 1998.

[45] K. Fulzele, D. J. DiGirolamo, Z. Liu, J. Xu, J. L. Messina, and T. L. Clemens, "Disruption of the insulin-like growth factor type 1 receptor in osteoblasts enhances insulin signaling and action," The Journal of Biological Chemistry, vol. 282, no. 35, pp. 2564925658, 2007.

[46] P. Bialek, B. Kern, X. Yang et al., "A twist code determines the onset of osteoblast differentiation," Developmental Cell, vol. 6, no. 3, pp. 423-435, 2004. 
[47] B. F. Boyce and L. Xing, "Functions of RANKL/RANK/OPG in bone modeling and remodeling," Archives of Biochemistry and Biophysics, vol. 473, no. 2, pp. 139-146, 2008.

[48] M. Pérez-Sayáns, J. M. Somoza-Martín, F. Barros-Angueira, J. M. G. Rey, and A. García-García, "RANK/RANKL/OPG role in distraction osteogenesis," Oral Surgery, Oral Medicine, Oral Pathology, Oral Radiology and Endodontology, vol.109, no. 5, pp. 679-686, 2010.

[49] T. Yoshizawa, "Diabetes mellitus and osteoporosis. Insulin signaling and bone/glucose/energy metabolism," Clinical Calcium, vol. 22, no. 9, pp. 1367-1373, 2012.

[50] S. Avnet, F. Perut, M. Salerno, L. Sciacca, and N. Baldini, "Insulin receptor isoforms are differently expressed during human osteoblastogenesis," Differentiation, vol. 83, no. 5, pp. 242-248, 2012.

[51] T. L. Clemens and G. Karsenty, "The osteoblast: an insulin target cell controlling glucose homeostasis," Journal of Bone and Mineral Research, vol. 26, no. 4, pp. 677-680, 2011.

[52] D. J. Walther, J.-U. Peter, S. Bashammakh et al., "Synthesis of serotonin by a second tryptophan hydroxylase isoform," Science, vol. 299, no. 5603, p. 76, 2003.

[53] V. K. Yadav, F. Oury, N. Suda et al., "A serotonin-dependent mechanism explains the leptin regulation of bone mass, appetite, and energy expenditure," Cell, vol. 138, no. 5, pp. 976989, 2009.

[54] C. J. Rosen, "Leptin's RIGHT turn to the brain stem," Cell Metabolism, vol. 10, no. 4, pp. 243-244, 2009.

[55] W. Cousin, A. Courseaux, A. Ladoux, C. Dani, and P. Peraldi, "Cloning of hOST-PTP: the only example of a protein-tyrosinephosphatase the function of which has been lost between rodent and human," Biochemical and Biophysical Research Communications, vol. 321, no. 1, pp. 259-265, 2004.

[56] P. Szulc and P. D. Delmas, "Biochemical markers of bone turnover: potential use in the investigation and management of postmenopausal osteoporosis," Osteoporosis International, vol. 19, no. 12, pp. 1683-1704, 2008.

[57] B. Merle and P. D. Delmas, "Normal carboxylation of circulating osteocalcin (bone Gla-protein) in Paget's disease of bone," Bone and Mineral, vol. 11, no. 2, pp. 237-245, 1990.

[58] L. J. Sokoll, S. L. Booth, K. W. Davidson, G. E. Dallal, and J. A. Sadowski, "Diurnal variation in total and undercarboxylated osteocalcin: Influence of increased dietary phylloquinone," Calcified Tissue International, vol. 62, no. 5, pp. 447-452, 1998.

[59] C. Rosenquist, P. Qvist, N. Bjarnason, and C. Christiansen, "Measurement of a more stable region of osteocalcin in serum by ELISA with two monoclonal antibodies," Clinical Chemistry, vol. 41, no. 10, pp. 1439-1445, 1995.

[60] P. Garnero, M. Grimaux, B. Demiaux, C. Preaudat, P. Seguin, and P. D. Delmas, "Measurement of serum osteocalcin with a human-specific two-site immunoradiometric assay," Journal of Bone and Mineral Research, vol. 7, no. 12, pp. 1389-1398, 1992.

[61] S. C. Corlett, M. Couch, A. D. Care, and A. R. Sykes, "Measurement of plasma osteocalcin in sheep: assessment of circadian variation, the effects of age and nutritional status and the response to perturbation of the adrenocortical axis," Experimental Physiology, vol. 75, no. 4, pp. 515-527, 1990.

[62] J. Nishimura, N. Arai, and J.-I. Tohmatsu, "Measurement of serum undercarboxylated osteocalcin by ECLIA with the "Picolumi ucOC" kit," Clinical calcium, vol. 17, no. 11, pp. 17021708, 2007.
[63] C. M. Gundberg, S. D. Nieman, S. Abrams, and H. Rosen, "Vitamin K status and bone health: an analysis of methods for determination of undercarboxylated osteocalcin," Journal of Clinical Endocrinology and Metabolism, vol. 83, no. 9, pp. 32583266, 1998.

[64] M. K. Shea, E. J. Benjamin, J. Dupuis et al., "Genetic and nongenetic correlates of vitamins $\mathrm{K}$ and D," European Journal of Clinical Nutrition, vol. 63, no. 4, pp. 458-464, 2009.

[65] P. Garnero, "Markers of bone turnover for the prediction of fracture risk," Osteoporosis International, vol. 11, no. 6, pp. S55S65, 2000.

[66] L. R. McCabe, "Understanding the pathology and mechanisms of type I diabetic bone loss," Journal of Cellular Biochemistry, vol. 102, no. 6, pp. 1343-1357, 2007.

[67] M. Inaba, Y. Nishizawa, K. Mita et al., "Poor glycemic control impairs the response of biochemical parameters of bone formation and resorption to exogenous 1,25-dihydroxyvitamin D3 in patients with type 2 diabetes," Osteoporosis International, vol. 9, no. 6, pp. 525-531, 1999.

[68] P. Gerdhem, A. Isaksson, K. Åkesson, and K. J. Obrant, "Increased bone density and decreased bone turnover, but no evident alteration of fracture susceptibility in elderly women with diabetes mellitus," Osteoporosis International, vol. 16, no. 12, pp. 1506-1512, 2005.

[69] J. M. Kindblom, C. Ohlsson, O. Ljunggren et al., "Plasma osteocalcin is inversely related to fat mass and plasma glucose in elderly Swedish men," Journal of Bone and Mineral Research, vol. 24, no. 5, pp. 785-791, 2009.

[70] E. F. Papakitsou, A. N. Margioris, K. E. Dretakis et al., "Body mass index (BMI) and parameters of bone formation and resorption in postmenopausal women," Maturitas, vol. 47, no. 3, pp. 185-193, 2004.

[71] P. Ravn, G. Cizza, N. H. Bjarnason et al., "Low body mass index is an important risk factor for low bone mass and increased bone loss in early postmenopausal women," Journal of Bone and Mineral Research, vol. 14, no. 9, pp. 1622-1627, 1999.

[72] I. Kanazawa, T. Yamaguchi, M. Yamauchi et al., "Serum undercarboxylated osteocalcin was inversely associated with plasma glucose level and fat mass in type 2 diabetes mellitus," Osteoporosis International, vol. 22, no. 1, pp. 187-194, 2011. 


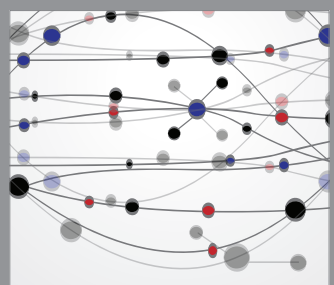

The Scientific World Journal
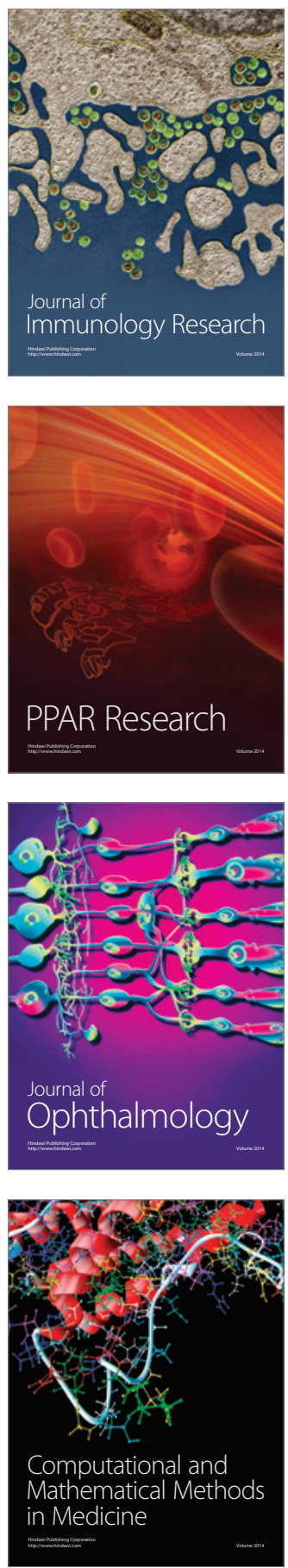

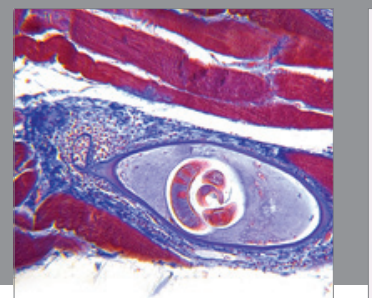

Gastroenterology

Research and Practice
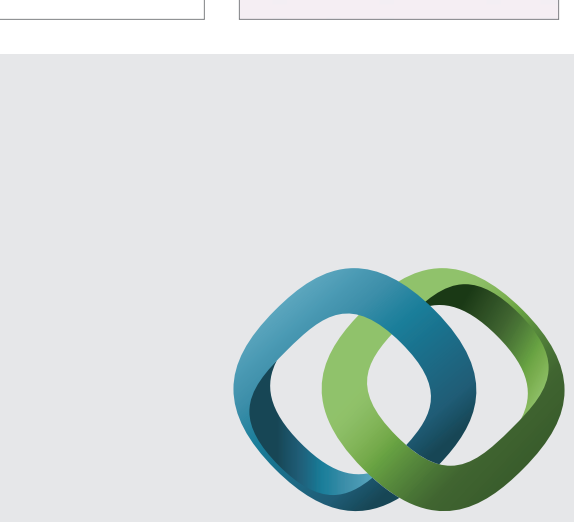

\section{Hindawi}

Submit your manuscripts at

http://www.hindawi.com
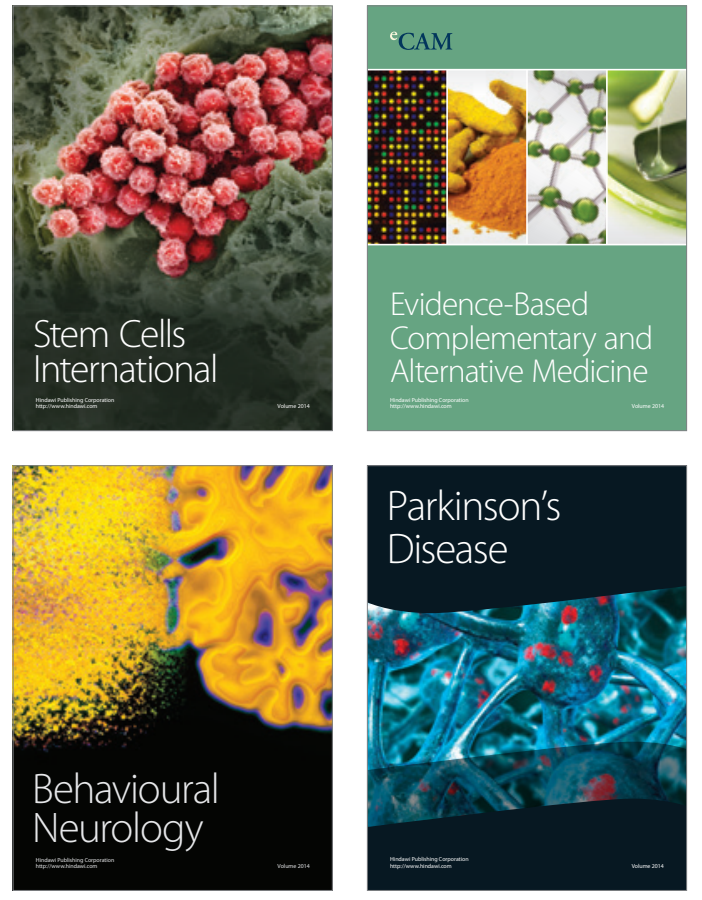
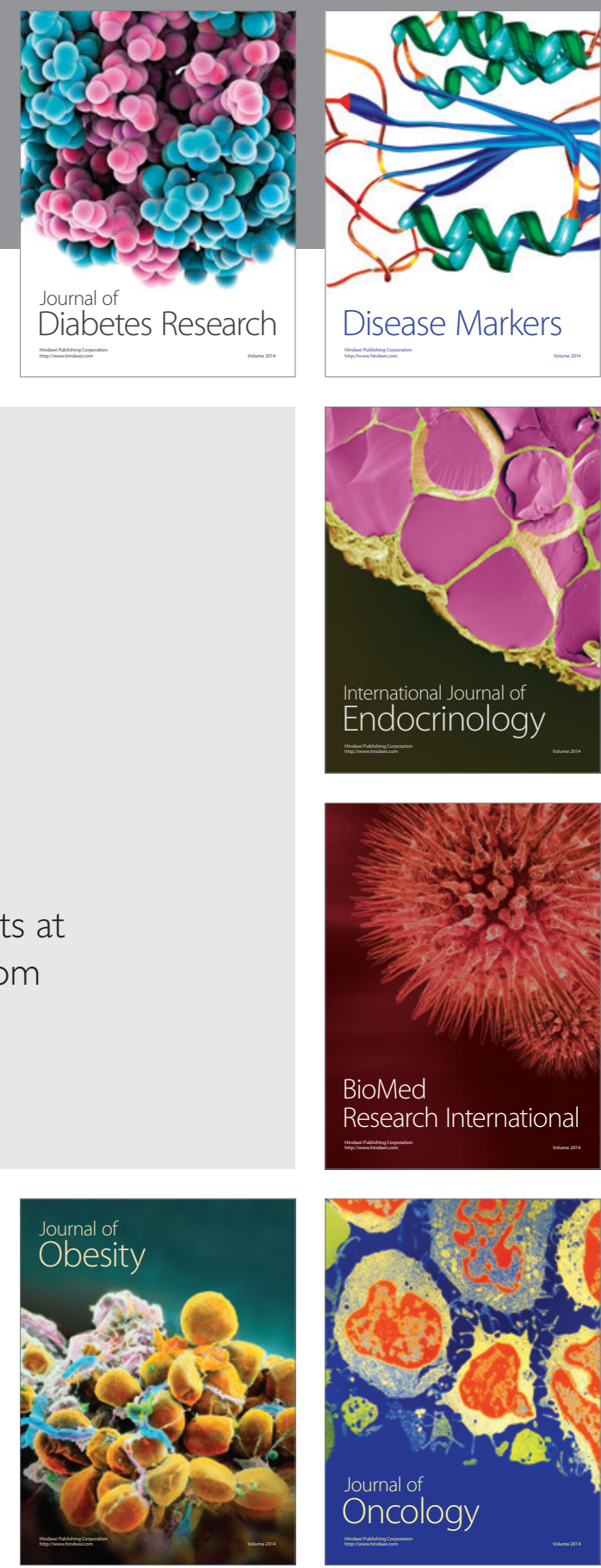

Disease Markers
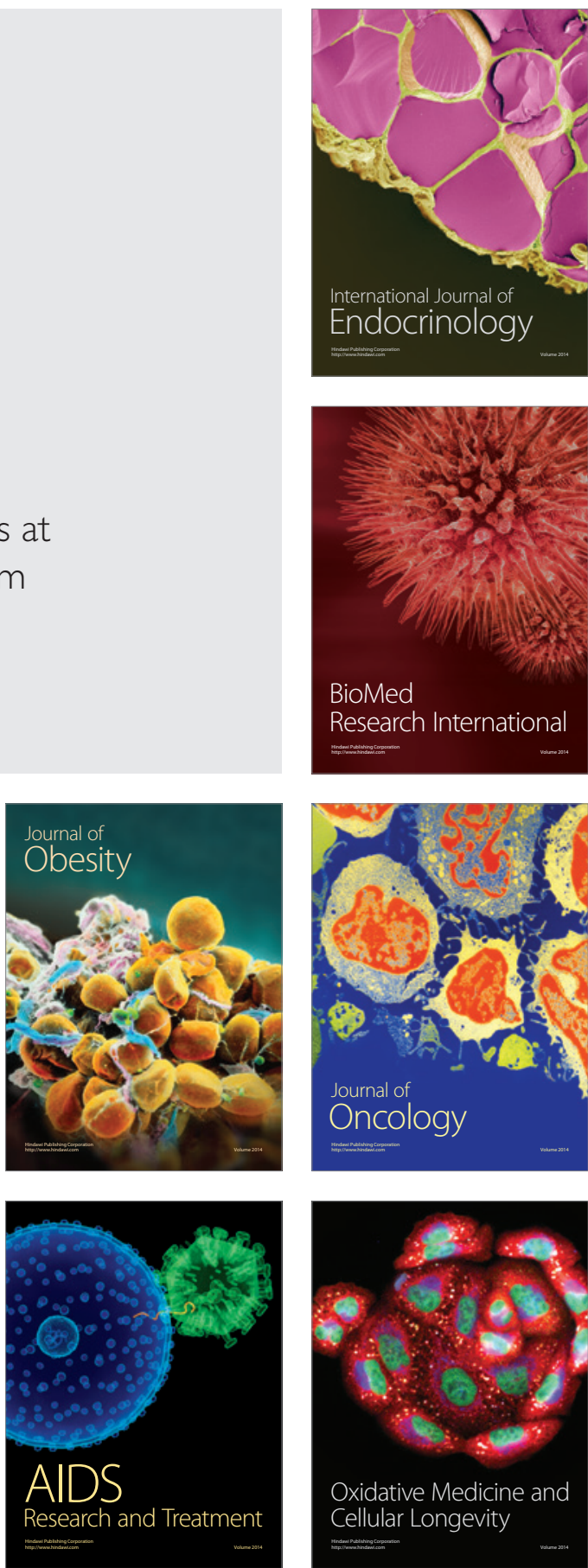